\title{
Anatomy in Patients with 22q11 Deletion and Pulmonary Atresia with Ventricular Septal Defect and Major Aortopulmonary Collaterals
}

\author{
Ashish O. Sureka ${ }^{1}$, Lynn F. Peng ${ }^{2}$, Olaf Reinhartz ${ }^{2}$, V. Mohan Reddy ${ }^{2}$, Frank L. Hanley ${ }^{2}$ \\ ${ }^{1}$ Pediatrix Cardiology of North Texas, Dallas, USA \\ ${ }^{2}$ Lucille Packard Children's Hospital, Palo Alto, USA \\ E-mail:ashish_sureka@pediatrix.com
}

Received April 30, 2011; revised June 20, 2011; accepted July 6, 2011

\begin{abstract}
s
We performed a retrospective analysis of patients with and without 22q11 deletion undergoing surgery for pulmonary atresia with ventricular septal defect and major aortopulmonary collaterals between January 2004 and August 2009 at our institutions. Information was collected on collateral origin, arch sidedness, presence of central pulmonary arteries, and presence of an aberrant subclavian vessel. While patients with 22q11 deletion were more likely to have collateral origin from brachiocephalic vessels, patients without 22q11 deletion were more likely to have collateral origin from the descending aorta. There was no significant difference in arch sidedness or the presence of central pulmonary arteries. Patients with 22q11 deletion were more likely to have an aberrant subclavian artery $(15 / 46$ vs 5/54, p < 0.05), whether a left or right arch was present. Nine of the fifteen 22q11 deletion patients had a collateral originating from an aberrant subclavian artery. In time, genomic and embryologic research may help determine the exact mechanisms by which 22q11 deletion contributes to the development of congenital heart disease such as pulmonary atresia with ventricular septal defect and major aortopulmonary collaterals.
\end{abstract}

Keywords: Tetralogy of Fallot with Pulmonary Atresia, Pulmonary Atresia with Ventricular Septal Defect, 22q11 Deletion

\section{Introduction}

The disease spectrum known as 22q11 deletion syndrome is associated with cardiac, immunologic and psychiatric abnormalities. Although the exact mechanism of the deletion's effect is unknown, abnormal neural crest cell migration seems to contribute [1]. The T-Box 1(TBX1) genetic pathway appears to be involved in the formation of the pharyngeal arches and the cardiac outflow tracts $[2,3]$. Mice heterozygous for the TBX1 deletion have a high frequency of defects of the fourth aortic arch [1,4]. In addition, haploinsufficiency of TBX1 appears to cause poor development of the distal outflow tracts [5-9].

A particularly challenging lesion common in these patients is pulmonary atresia with ventricular septal defect with major aortopulmonary collaterals (PA/VSD/ MAPCA's), also known as tetralogy of Fallot with pulmonary atresia and major aortopulmonary collaterals. Among patients with this lesion, several studies have attempted to highlight the differences between the patients with and without 22q11 deletion. In one study comparing 23 cases to controls, patients with the deletion were more likely to have a right aortic arch, aberrant subclavian artery, and MAPCA's than patients without the deletion. There was no difference in the incidence of absent true confluent central pulmonary arteries [10].

A prospective study describing 25 patients compared to controls found those with PA/VSD and 22q11 deletion were more likely to have MAPCA's and absent confluent central pulmonary arteries than those without the deletion. There was no difference in the incidence of an aberrant subclavian artery. Among the 25 patients, ten had MAPCA's, all originating from the descending aorta [11].

This study sought to examine the comparative anatomy in patients with pulmonary atresia with ventricular septal defect with major aortopulmonary collaterals with and without 22q11 deletion. 


\section{Materials and Methods}

We reviewed the data for all patients with PA/VSD/ MAPCA's who underwent surgery at our institutions between January 2004 and August 2009. All patients underwent cardiac catheterization and echocardiogram before operation; in some cases, the catheterization was performed at outside institutions. All angiograms were reviewed by the investigators at our institution. All catheterizations were performed systematically to establish the following information:

a) collateral origin(s), whether from brachiocephalic arterial vessels, ductus-like collateral vessels, descending aorta, or coronary arteries (patients often have multiple collaterals from various origins)

b) presence of central pulmonary arteries

c) arch sidedness

d) presence of an aberrant subclavian artery

Patients without selective angiograms that adequately defined collateral origin or arch sidedness were excluded from the study. Collaterals originating from the undersurface of the aorta but not encircled by the recurrent laryngeal nerve were termed 'ductus-like collateral vessels,' while other collaterals originated from brachiocephalic arteries, the descending aorta, or coronary arteries.

All patients underwent FISH testing for 22q11 deletion. Many patients were referred; the method of 22q11 flourescent in situ hybridization (FISH) testing varied by referring institution.

Statistical analysis was performed by Fisher's exact test. Analysis was performed with SAS version 9.1 (SAS Institutes Inc., Cary, NC). Significance was determined at a $\mathrm{p}$ value of less than 0.05 . All $\mathrm{p}$ values were twotailed.

\section{Results}

Table 1 outlines the anatomical differences of PA/VSD /MAPCA's patients with 22q11 deletion compared to the patients without the deletion. Patients with 22q11 deletion were significantly more likely to have an aberrant subclavian artery, regardless of arch sidedness ( $<<0.05$ ). Among the 15 patients with an aberrant subclavian artery, 6 had a left aortic arch with an aberrant right subclavian artery, while 9 had a right aortic arch with an aberrant left subclavian artery. Nine of the 15 patients with an aberrant subclavian artery (60\%) had a collateral originating from that vessel.

Patients with 22q11 deletion were significantly more likely to have a collateral vessel arise from a brachiocephalic artery $(\mathrm{p}<0.01)$. Patients without 22q11 deletion were more likely to have a collateral vessel arising from the descending aorta $(\mathrm{p}<0.05)$.
Table 1. Anatomical differences in PA/VSD/MAPCA's patients with and without 22q11 deletion.

\begin{tabular}{|c|c|c|c|}
\hline Characteristic & 22q11 Positive & 22q11 Negative & p-value \\
\hline $\begin{array}{l}\text { Brachiocephalic } \\
\text { artery collateral } \\
\text { vessels }\end{array}$ & 25 of $46(54 \%)$ & 13 of $54(24 \%)$ & $0.035^{*}$ \\
\hline $\begin{array}{c}\text { Ductus_like } \\
\text { collateral vessels }\end{array}$ & 7 of $46(15 \%)$ & 2 of $54(4 \%)$ & 0.076 \\
\hline $\begin{array}{l}\text { Descending aorta } \\
\text { collateral vessels }\end{array}$ & 40 of $46(87 \%)$ & 53 of $54(98 \%)$ & $0.046^{*}$ \\
\hline $\begin{array}{l}\text { Coronary arter y } \\
\text { collateral vessels }\end{array}$ & 2 of $46(4 \%)$ & 4 of $54(7 \%)$ & 0.68 \\
\hline $\begin{array}{l}\text { Presence of central } \\
\text { pulmonary arteries }\end{array}$ & 35 of $46(76 \%)$ & 45 of $54(83 \%)$ & 0.45 \\
\hline $\begin{array}{l}\text { Presence of right } \\
\text { aortic arch }\end{array}$ & 20 of $46(43 \%)$ & 21 of $54(39 \%)$ & 0.69 \\
\hline $\begin{array}{c}\text { Presence of aberrant } \\
\text { subclavian artery }\end{array}$ & 15 of 46 (33\%) & 5 of $54(9 \%)$ & $0.0052 *$ \\
\hline
\end{tabular}

\section{Discussion}

To our knowledge, this is the first report detailing the origin of collateral vessels in PA/VSD/MAPCA's with and without 22q11 deletion. As in previous reports, 22q11 deletion was associated with an aberrant subclavian artery $[12,13]$. Notably, in our study the presence of the 22q11 deletion was not significantly predictive for a right aortic arch among patients with PA/VSD/ MAPCA's.

These findings could help guide diagnostic testing in patients with PA/VSD/MAPCA's, with and without 22q11 deletion. Testing for sources of collateral circulation is generally not methodical-the aorta, coronary arteries, and brachiocephalic vessels are assessed by catheterization. In the era of widespread availability of FISH testing, we believe our findings may help direct assessment for collaterals and abnormalities of vessel branching based on the patient's 22q11 status. Patients with 22q11 deletion are more likely to have collaterals from brachiocephalic vessels and an aberrant subclavian artery, regardless of arch sidedness.

The results of this study may also have long-term implications for determining the mechanism by which 22q11 deletion interferes with the normal development of the cardiac outflow tracts. Though abnormal migration of neural crest-derived tissues has been implicated, it is unclear why and how patients with the deletion would more often develop collateral circulation from brachiocephalic vessels. More precise molecular characterization could be helpful.

This study had several limitations. Referral bias was a significant concern. As our institution is a referral center for PA/VSD/MAPCA's, our patients may not have 
anatomy representative of the entire population of PA/ VSD/MAPCA's patients, with and without 22q11 deletion. Also, the fact that some of the patients had studies (including the FISH test and catheterization) at various institutions introduces bias.

Collaterals can regress over time, and these patients entered our system at various ages. A cohort study of all newborns may yield different results.

Studies with more PA/VSD/MAPCA's patients would be helpful to better characterize the anatomy in those with and without 22q11 deletion. In time, genomic and embryologic research may help determine the exact mechanisms by which 22q11 deletion contributes to the development of congenital heart disease such as PA/VSD /MAPCA's.

\section{References}

[1] L. A. Jerome and V. E. Papaioannou, "DiGeorge Syndrome Phenotype in Mice Mutant for the T-Box Gene, Tbx1,” Nature Genetics, Vol. 27, No. 3, 2001, pp. 286-291. doi:10.1038/85845

[2] A. Baldini, "DiGeorge Syndrome: An Update," Current Opinion in Cardiology, Vol. 19, No. 3, 2004, pp. 201-204. doi:10.1097/00001573-200405000-00002

[3] A. Baldini, "Dissecting Contiguous Gene Defects: TBX1," Current Opinion in Genetics \& Development, Vol. 15, No. 3, 2005, pp 279-284. doi:10.1016/j.gde.2005.03.001

[4] E. A. Lindsay, F. Vitelli, et al, "Tbx1 Haploinsufficieny in the DiGeorge Syndrome Region Causes Aortic Arch Defects in Mice,” Nature, Vol. 410, No. 6824, 2001, pp. 97-101. doi:10.1038/35065105

[5] T. Hu, H. Yamagishi, et al. "Tbx1 Regulates Fibroblast Growth Factors in the Anterior Heart Field through a Rein Forcing Autoregulatory Loop Involving Forkhead Transcrition Factors," Development, Vol. 131, No. 21, 2004, pp. 5491-502. doi:10.1242/dev.01399
[6] R. G. Kelly and V. E. Papaioannou, "Visualization of Outflow Tract Development in the Absence of Tbx1 Using an FgF10 Enhancer Trap Transgene,” Developmental Dynamics, Vol. 236, No. 3, 2007, pp. 821-828. doi:10.1002/dvdy.21063

[7] F. Vitelli, M. Morishima, et al, “Tbx1 Mutation Causes Multiple Cardiovascular Defects and Disrupts Neural Crest and Cranial Nerve Migratory Pathways," Human Molecular Genetics, Vol. 11, No. 8, 2002, pp. 915-922. doi:10.1093/hmg/11.8.915

[8] H. Xu, M. Morishima, et al, “Tbx1 has a Dual Role in the Morphogenesis of the Cardiac Outflow Tract," Development, Vol. 131, No. 13, 2004, pp. 3217-3227. doi:10.1242/dev.01174

[9] Z. Zhang, T. Huynh, et al, "Mesodermal Expression of Tbx1 is Necessary and Sufficient for Pharyngeal Arch and Cardiac Outflow Tract Development,” Development Vol. 133, No. 18, 2006, pp. 3587-3595. doi:10.1242/dev.02539

[10] K. Momma, C. Kondo, et al, "Tetralogy of Fallot with Pulmonary Atresia Associated with Chromosome 22q11 Deletion," Journal of the American College of Cardiology, Vol. 27, No. 1, 1996, pp. 198-202. doi:10.1016/0735-1097(95)00415-7

[11] M. Chessa, G. Butera, et al, "Relation of Genotype 22q11 Deletion to Phenotype of Pulmonary Vessels in Tetralogy of Fallot and Pulmonary Atresia-Ventricular Septal Defect,” Heart, Vol. 79, No. 2, 1998, pp. 186-190.

[12] D. B. McElhinney, B. J. Clark, et al, "Association of Chromosome 22q11 Deletion with Isolated Anomalies of Aortic Arch Laterality and Branching," Journal of the American College of Cardiology, Vol. 37, No. 8, 2001, pp. 2114-2119. doi:10.1016/S0735-1097(01)01286-4

[13] R. Rauch, A. Rauch, et al, "Laterality of the Aortic Arch and Anomalies of the Subclavian Artery-Reliable Indicators for 22q11.2 Deletion Syndromes?” European Journal of Pediatrics, Vol. 163, No. 11, 2004, pp. 642-645. 